\title{
Expansion of tumor-infiltrating lymphocytes with substantial stem cell properties from vulvar cancer
}

\author{
Tingting Yao et al. ${ }^{1}$ and Yarong Liu ${ }^{1}$ \\ ${ }^{1}$ Affiliation not available
}

September 13, 2021

\begin{abstract}
Tumor-infiltrating lymphocyte (TIL) therapy has been clinically proved as a promising therapeutic approach for patients with solid tumor. TIL therapy could effectively control tumor growth in cervical cancer as indicated by a phase 2 pivotal trial with an objective response rate of $44.4 \%$. Vulvar cancer is believed to share a similar biological and immunological phenotype with cervical cancer. However, the therapeutic potential of TIL in vulvar cancer remains to be explored. In this study, we described a manufacturing procedure that can expand clinical-scale TILs from both vulvar cancer and cervical cancer with a high success rate. Characterization of the phenotype of TIL populations showed that TILs from vulvar cancer are prone to maintaining a higher percentage of progenitor-like phenotype and have stronger tumor-killing capacity compared to TILs from cervical cancer. TCR clonality analysis indicated that all TIL samples have more enriched TCR clones than PBMC, which might be expanded during anti-tumor responses and tend to be patient specific. Thus, our study provides a feasible method of TIL preparation from and a potential new therapeutic strategy for vulvar cancer patients.
\end{abstract}

Tingting Yao ${ }^{1,2}$, Zixiao $\mathrm{Shi}^{3}$, Jingwei Sun ${ }^{3}$, Yuhan Wang ${ }^{1,2}$, Haijian Zhou ${ }^{1,2}$, Yanru Chen ${ }^{4,5}$, Di Zhou ${ }^{3}$, Zhongqiu Lin ${ }^{1,2 \#}$, Yarong Liu ${ }^{3 \#}$

${ }^{1}$ Department of Gynecological Oncology, Sun Yat-sen Memorial Hospital, SunYat-sen University, 510120 Guangzhou, P.R. China

${ }^{2}$ Guangdong Provincial Key Laboratory of Malignant Tumor Epigenetics and Gene Regulation, Sun Yat-Sen Memorial Hospital, Sun Yat-Sen University, 510120 Guangzhou, P.R. China

${ }^{3}$ Grit Biotechnology Co., Ltd. Shanghai, 201210 Shanghai, P.R. China

${ }^{4}$ Children's Hospital of Fudan University at Xiamen, 361006 Xiamen, P.R. China

${ }^{5}$ Xiamen Children's Hospital, 361006 Xiamen, P.R. China

\# Correspondence:

Yarong Liu, Grit Biotechnology Co., Ltd. Shanghai, 1077 Zhangheng Road, PuDong, Shanghai, 201210, P.R. China

E-mail: yarong.liu@grit-bio.com

Zhongqiu Lin, Department of Gynecological Oncology, Sun Yat-sen Memorial Hospital, SunYat-sen University, 107 Yan Jiang West Road, 510120 Guangzhou, P.R. China, linzhongqiu@hotmail.com

\section{Key words}

Tumor infiltrating lymphocytes; immunotherapy; vulvar cancer; cervical cancer; stemness

\section{Introduction}


Vulvar cancer is a malignant tumor that originates from the vulvar, representing around $5 \%$ of all gynecological malignancies. The overall incidence and mobility of vulvar cancer has been rising in the past few decades(1). Nearly $90 \%$ of vulvar cancer cases are squamous cell carcinoma (SCC), followed by melanoma. So far, only limited treatment options are available for vulvar cancer. Surgery is still the major treatment strategy for vulvar cancer patients, sometimes with additional support of chemotherapy or radiotherapy. For vulvar cancer patients with regional and distant metastasis, the five-year survival rate of traditional therapies is $53 \%$ and $19 \%$, respectively $(2)$. Therefore, it is urgent to develop new therapeutic strategies for vulvar cancer patients.

Cancer immunotherapy, as represented by anti-PD1/PDL1 antibodies and CAR-T therapy, has revolutionized the history of cancer treatment and significantly prolonged the survival of cancer patients, especially patients with advanced or metastatic tumors $(3,4)$. However, only limited randomized clinical trials of immunotherapy have been performed for vulvar cancer patients(5). Immunohistochemistry analysis showed that considerable immune cell infiltration could be found in vulvar tumor $(6,7)$. Notably, $78 \%$ of HPVinduced SCC has shown high intraepithelial T cell infiltration and 40-60\% in HPV negative SCC. Moreover, a high intraepithelial infiltration with CD3 $+\mathrm{T}$ cells is associated with overall and recurrence-free survival(7), providing a solid evidence to consider developing immunotherapy for vulvar cancer.

TIL therapy is a type of adoptive cellular therapy, which collects infiltrated lymphocytes from the tumor through biopsy or surgery, amplifies them in the laboratory with interleukin-2 (IL-2) and then reinfuses back intravenously to the patient(8). Compared with CAR-T cell therapy, which significantly enhances the treatment of hematological malignancies, TIL therapy holds unique advantages in tackling the solid tumors. TILs collected from the tumor site have multiple TCR clones that can recognize heterogeneous tumor antigens, while CAR-T cells only recognize single antigen epitopes. Having been stimulated by tumor antigens in vivo, TILs usually express chemokine receptors on the surface and show superior homing capacity to tumor site upon adoptive transfer $(9,10)$. In addition, off-target toxicity is seldomly reported in TIL therapy.

The National Cancer Institute of the United States started to explore TIL therapy to treat patients with metastatic melanoma in 1988, and demonstrated an objective response rate (ORR) of $60 \%(11)$. For a long time, however, TIL therapy was only successful in treating melanoma. Currently, TIL preparation is being explored for more cancer types, such as non-small cell lung cancer, ovarian cancer, colorectal cancer, bile duct cancer, breast cancer and cervical cancer, and such TILs have shown promising results in clinical trials(1214). A recent phase 2 trial of autologous TIL therapy showed an ORR of $44.4 \%$ in 27 cervical cancer patients who had received multiple lines of conventional treatment(15).

The relatively high $\mathrm{T}$ cell infiltration found in the tumor site indicates the high potential of TIL as a new therapeutic strategy for patients with vulvar cancer, but the feasibility of TIL collection and amplification from vulvar cancer hasn't been investigated yet. Therefore, we isolated and expanded TILs from vulvar cancer (VC TILs) in comparison with those from cervical cancer (CC TILs) in the present study. The TCR diversity and clonetypes of TIL samples were further compared with PBMC (peripheral blood mononuclear cell) from the same donor. By evaluating and comparing multiple indicators of TILs from these two tumor types, we demonstrated that our manufacturing process can successfully culture and expand VC TILs with good effector functions, and both VC and CC TILs have more enriched TCR clones than PBMC.

\section{Materials and methods}

\section{Patient subjects}

Participants were female patients between 18 and 70 years old with at least one tumor lesion with a diameter of more than $1.5 \mathrm{~cm}$, and could be treated with reasonable tumor reduction surgery in accordance with NCCN clinical guidelines. Patients who received radiotherapy within 28 days before surgery were excluded. Tumor samples in situ were obtained by hysterectomy, and superficial lymph node metastases were obtained by minimally invasive surgery. This study was approved by the Medical Ethics Committee of Sun Yat-sen Memorial Hospital of Sun Yat-sen University, with the Approval number SYSEC-KY-KS-2019-147. 


\section{TIL preparation}

Primary tumors or lymph node metastases were separated into 1-20mm3 fragments and placed in a Grex or gas permissible bag. In preREP phase, the tissues were expanded with timely replenished or replaced medium for about two weeks. Culture medium contained 3000IU/mL rhIL-2 (T\&L Biotechnology). In the following REP phase, TILs were stimulated with anti-human CD3 antibody (OKT3, T\&L Biotechnology) in the presence of irradiated feeder cells and then expanded for about two weeks.

\section{Flow cytometry}

Expanded TIL was stained with fluorescent antibodies for human CD3 (BD, 564713), CD4 (BD, 557852), CD8 (BD, 564526), CD56 (BD, 555516), CD28 (BD, 555730), CD103 (Biolegend, 350216), CD39 (BD, 563679), TCF1 (Biolegend, 655208), PD-1 (BD, 564323), TIM-3 (BD, 565558), LAG-3 (BD, 565720), CD45RA (BD, 562885), CCR7 (BD, 565867). All cells were stained with a Live/Dead viability dye (eBioscience, 65-086514). For intracellular staining of TCF1, cells were fixed with Transcription Factor Buffer Set (BD, 562574), and then TCF1 was stained in accordance with the manufacturer's protocol (Biolegend). Data were acquired on an MACSQuant Analyzer 10 flow cytometer (Miltenyi) and analyzed using FlowJo software (Treestar).

\section{Functional assay}

For CD107a expression analysis, TILs were seeded in a 96-well plate as 1e5/well, and three duplicated wells were set for each group. TransAct (Miltenyi) was added to stimulate TILs overnight. Then, CD107a was analyzed by flow cytometry on the next day. For cytokine secretion and cytotoxicity analysis, HeLa cells labeled with CFSE were seeded in a 96-well plate as 5e4/well with 1.5e5/well TILs. The supernatant was collected at $18 \mathrm{hrs}$ and cytokine production was analyzed by CBA (BD). Then, DAPI was added into the co-culture system to analyze the tumor killing by detecting dead cells with flow cytometry.

\section{RNA Isolation and TCR Sequencing}

PBMCs were separated by density gradient centrifugation. Total RNA was extracted by QIAampß RNA Blood Mini Kits (QIAGEN, USA) according to the manufacturer's instructions. TCR $\alpha / \beta$ libraries for sequencing were constructed with the SMARTer Human TCR a/b Profiling Kit (TAKARA, USA) following the manufacturer's protocol. First-strand cDNA synthesis from RNA was primed by the TCR dT Primer and used the SMART-Seq v4 Oligonucleotide for template switching at the 5' end of the transcript. Following reverse transcription, two rounds of PCR were performed in succession to amplify cDNA sequences corresponding to variable regions of TCR- $\alpha$ and TCR- $\beta$ transcripts and add the sequencing adapter and sample index. The PCR product was then purified with magnetic beads. The libraries were pooled and sequenced on an Illumina MiSeq sequencer (Illumina, USA) with paired-end, $2 \times 300$ base pair reads. The used TCR gene regions were determined based on the reference sequences from the Immunogenetics (IMGT) database (V 3.1.34, http://www.imgt.org). All TCR gene segments were determined by MiXCR(16).

\section{Statistical analysis}

Statistical analyses were performed using Prism 7.0 (GraphPad Software) and R 4.1.0. Bar or dot graphs indicate means \pm SEM. Non-paired t test (two-tailed) was used for the comparison between CC and VC datasets. datasets. $\mathrm{P}$ values are marked in the corresponding figures and two side $\mathrm{p}$ values $<0.05$ were considered as statistically significant. Vegan package in $\mathrm{R}$ software were utilized to calculate Shannon-Weaver index of TCR CDR3 sequence.

\section{Results}

\section{Demographics of patient samples}

Primary cervical and vulvar tumors or tumor metastases in lymph node (LN) obtained from surgical specimens were collected from 10 cervical cancer patients and 4 vulvar cancer patients, and all were successfully prepared. The feasibility of TIL expansion was evaluated in these two tumor types. Sample information is summarized in Table I. 


\section{Expansion of TIL from vulvar and cervical tumors}

VC TILs could be successfully expanded using the same procedure as that for CC TILs. Briefly, after the acquisition and transportation of tumor surgical specimens to our manufacturing site, TIL cells were collected from the tumor fragments, cultured in a previous rapid expansion phase (preREP), and then amplified in a rapid expansion phase (REP). Expanded TIL cells were then cryopreserved and transported to the clinical center (Figure 1A). The average cell number in preREP from the four batches of VC TILs is greater than $2 \times 107$ (Figure 1B), and the average expansion fold in REP is greater than 1000 (Figure 1C), which is higher than that of CC TILs. In general, VC TILs can be successfully cultured and amplified in vitro.

\section{VC TILs demonstrated immune compositions and T cell phenotype similar to those of CC TILs}

To characterize the components and phenotype of expanded VC TILs, we analyzed the immune subsets as well as markers involved in T cell activation, differentiation and function. At the end of preREP, all TIL samples are mainly composed of CD3+CD56- T cells (mean>50\%) with a small fragment of CD3-CD56+ NK cells (around 10\%) (Figure 2A). The percentage of CD8+ and CD4+ T cells showed high variance among different individuals in both cancer types (Figure 2B). Both VC and CC TILs showed high expression of CD28, a marker associated with $\mathrm{T}$ cell activation and antitumor function(17), suggesting a functional phenotype of TILs in preREP with our preparation procedure (Figure 2C, 2D).

At the end of REP, T cells were further expanded in culture (most $>90 \%$ ) and the NK population decreased to a minimal level (Figure 3A). CD8+ or CD4+ subset in the $\mathrm{T}$ cell population still can still be observed in several samples in both types of cancers (Figure 3B). Similar to TILs in the preREP, the CD28+ T cells remained at a high percentage in the REP, while CD28+CD8+ T cells in VC TILs showed an even higher percentage compared to CC TILs (Figure 3C). We further analyzed the expression of inhibitory receptors TIM-3 and LAG-3, both of which are involved in T cell exhaustion on TILs(18). Both CC and VC TILs highly expressed TIM-3, but VC TILs showed lower expression of LAG-3 on both CD8+ and CD4+ T cell populations (Figure 3D). Tregs in both VC and CC TILs are under 5\% (Figure 3E). Taken together, both VC and CC TILs are predominantly composed of T cells at the end of REP, but VC TILs may have a more active CD8+ $\mathrm{T}$ cell population as indicated by higher CD28 and lower LAG-3 expression (Figure 3F).

\section{VC TILs may have higher stem-like property than CC TILs}

A recent study found that tumor-infiltrating CD8+ T cells can be divided into two functionally distinct subgroups: a terminally differentiated group marked by high expression of immune-checkpoint molecules such as PD-1 and TIM-3, and a stem-like group with low checkpoints but high TCF1 and CD28 expression(19). PD-1 (programmed death receptor 1, also known as CD279) is a co-inhibitory receptor restraining T cell function and anti-tumor response, thus considered as a marker of T cell exhaustion(20). TCF1 is a key transcription factor in stem cell-like $\mathrm{T}$ cells, and it plays a critical role in sustaining immune response to tumor and chronic virus infection $(21,22)$. To better characterize the functional profile of TILs after the REP process, we analyzed the expression of PD-1 and TCF1 in VC and CC TILs. The percentage of PD-1 positive CD4+ and CD8+ T cells in CC TILs is only around 10\%, but it is even lower in VC TILs (Figure 4A). Almost $100 \%$ of CD8+ VC TILs is TCF1+, which is significantly higher than that in CC TILs (Figure 4B), indicating that VC TILs may possess a stronger stem-like property and, thus, a better potential to amplify and survive in vivo than CC TILs. We further examined the memory phenotype of VC and CC TILs by CD45RA and CCR7 staining and found that VC TILs have a higher percentage of naïve and central memory $\mathrm{T}$ cells $(\mathrm{Tcm})$ (Figure 4C) and a lower percentage of effector memory and effector memory RA T cells (Tem and TemRA) (Figure 4D) than CC TILs, especially in CD4+ T cells, which is consistent with the higher stem-like phenotype of VC TILs.

In addition, we profiled our TIL samples by CD103 and CD39 staining as CD103+CD39+ tumor-infiltrating CD8 + T cells that may represent tumor-reactive cells in solid tumors and are associated with better overall survival(23). We observed that 6/10 of CC TIL samples and 2/4 of VC TIL samples have more than $20 \%$ CD8+ T cells showing CD103 and CD39 double positivity, but this ratio is low in CD4+ T cells (Figure 
$4 \mathrm{E})$.

\section{uCDR3 number and diversity characterization}

The number of unique CDR3 (uCDR3) in 8 samples including both VC and CC samples was determined by CDR3 Nucleic acid sequence, and the number of uCDR3 between PBMC and TIL was compared. The number of uCDR3 in TIL (mean $=14122.38$, median $=8087$ ) was significantly lower than that of PBMC $($ mean $=63679.62$, median $=56350.5)$ (Figure $5 \mathrm{~A}, \mathrm{P}=0.00133)$. In addition, the number of the uCDR3 in each TIL sample was lower than that in its paired PBMC sample (Figure 5B). The accumulate relative abundance of Top10 ranked uCDR3 in every TIL sample (mean $=50.49$, median $=49.83)$ is higher than that in the paired PBMC sample $($ mean $=16.32$, median $=13.72)$. Notably, the accumulate relative abundance of Top10 uCDR3 is more than $50 \%$ in 4 out of 8 TIL samples. Moreover, we analyzed the Shannon Entropy in each sample, which is a quantitative measure of uncertainty that simultaneously takes both richness and evenness into account. A higher Shannon entropy indicates that the TCR sample has more types of uCDR3 or a more even distribution of CDR3 reads. Shannon Entropy of TIL (mean $=4.93$, median $=4.96)$ was significantly lower than that of PBMC (mean $=9.01$, median $=9.31)$ (Figure $5 \mathrm{D}, \mathrm{P}=0.0004)$. The Shannon Entropy of each TIL sample was lower than that in its paired PBMC sample (Figure 5E). Collectively, the comparison of the uCDR3 number, relative abundance distribution of Top10 ranked uCDR3 and Shannon Entropy suggests that TIL samples have more enriched T cell clones than PBMC.

\section{Counts and reads fraction distribution of TCR clonotypes}

A total of 107732 clonotypes were identified in all TIL samples. Detection times of each clonotypes in TIL samples were summarized in Figure 6A. Most clonotypes (98.467\%) were detected in only one TIL sample. Only $1.53 \%$ clonotypes were found in more than one TIL sample and $0.01 \%$ were found in 4 or more samples, suggesting that the repertoire of TIL TCR were patient-specific (Figure 6A). Reads of most shared clonotypes represented low fraction, with $77.8 \%$ of clonotypes accounting for less than $0.001 \%$ reads fraction and $18.2 \%$ clonotypes accounting for 0.001\%-0.01\% (Figure 6B).

\section{Functional analysis of CC and VC TILs}

To investigate whether the expanded TILs are responsive to extracellular restimulation, VC and CC TILs are restimulated overnight by $\mathrm{T}$ cell activator. Upregulation of CD107a, a marker of degranulation indicating T cell cytotoxic activity, can be observed on a portion of VC and CC TILs (Figure 7A). We further analyzed the cytokine production of TILs in response to stimulation of HeLa cells, a cervical cancer cell line, and found that both CC and VC TILs produced high levels of IFN- $\gamma$ and TNF- $\alpha$, and that VC TILs tended to release more of these two cytokines (Figure 7B and C). Finally, the cytotoxicity potential of TILs was examined by HeLa cell lysis in a co-culture system. VC TILs tended to show higher killing of HeLa cells compared to CC TILs, suggesting the stronger tumor killing potential of VC TILs (Figure 7D).

\section{Discussion}

Cancer immunotherapy harnessing the immune system of patients to attack tumors has achieved promising clinical benefits in patients with advanced cancers. However, most tumors, especially solid tumors, remain largely unresponsive to current therapies(24), thus leaving still unmet demands in the clinic. TIL is a type of adoptive cellular therapy with distinctive advantages in the treatment of solid tumors, including but not limited to its diverse TCR clones capable of recognizing heterogeneous tumor antigens, its superior homing ability to the tumor site and low off-target toxicity. So far, TIL therapy has shown promising clinical results in several types of solid tumors, such as metastatic melanoma and cervical cancer. Vulvar cancer is a serious gynecological malignancy with increasing incidence in the last few decades. Effective therapeutic strategy is still an urgent need, especially for advanced vulvar cancer. Lymphocyte infiltration in the tumor site, together with its positive association with overall and recurrence-free survival, provides a solid theoretical and practical foundation for applying TIL therapy to vulvar cancer patients. Therefore, we explored the feasibility of isolating and expanding TILs in vitro for vulvar cancer.

We used a manufacturing process with optimized culture conditions based on previous TIL studies, and we 
have successfully produced CC TILs displaying desirable properties for therapeutic uses. In this study, we showed that VC TILs can be amplified from both in situ tumor samples and lymph node metastases. Even for samples with a low percentage of $\mathrm{T}$ cells in the preREP stage, it should be noted that VC TILs can still be successfully amplified in subsequent expansion with around 95\% CD3+ T cells in the final product. Although $\mathrm{T}$ cells in the tumor environment usually showed an exhausted phenotype with attenuated functions, the high CD28 and low PD-1 expression on VC and CC TILs after REP indicated that TILs may be able to mount antitumor activities in response to tumor antigens. Besides, the percentage of Tregs in the final product is very low (below $5 \%$ percent).

The uCDR3 number and diversity analysis revealed the expansion and enrichment of certain TCR clonotypes in the TIL product. The relative abundance of Top10 and Top100 ranked uCDR3 in TILs is much higher than that in the PBMC samples. Most clonotypes were detected only in one sample, and most shared clonotypes were clones with low frequency, suggesting that most of the TCR repertoires in TIL product are patient-specific. As reported by Bobisse et al., this patient-specific TCR can be identified even in some patients with tumors harboring a relatively low mutational load such as ovarian cancer. The patient-specific TCR may be generated by differential expression of tumor antigens, such as the expression of patient-specific neoantigens(25) or patient-specific responding process against autologous tumor(26).

Functional analysis showed that our TIL product, especially VC TILs, can upregulate CD107a and produce IFN- $\gamma$ and TNF- $\alpha$ upon tumor or antibody stimulation and mediate cytotoxicity to HeLa cells. The phenotype and function of our TIL product is similar to those of TIL from melanoma as reported before(27), indicating that our cell production process is able to enrich effector T cells with antitumor responses. A major challenge to evaluate the tumor specificity of TIL is the availability of autologous tumor samples. Currently, we are developing methods to culture patient-derived cancer cells (PDCs) and organoids from vulvar cancer samples in vitro, which could be used to test the specific responses of VC TILs to vulvar tumor antigens in the future.

Compared with cervical cancer, the number of cells obtained from vulvar cancer samples after preREP is similar, but the average amplification rate during the REP stage is twice that of cervical cancer, demonstrating the good amplification capacity of VC TILs in culture. VC and CC TILs have similar T and NK cell composition, but the expression of activation marker CD28 and stem cell marker TCF1 is higher on VC TILs. Moreover, VC TILs showed better cytokine secretion and tumor-killing capability compared with that of CC TILs, indicating better effector function of VC TILs. Additional studies, such as profiling of T cell receptor repertoire and analysis of TCR specificity to shared tumor antigens or neoantigens, will facilitate a better understanding of VC TILs.

Taken together, we have demonstrated in this report that TILs can be successfully harvested and expanded from tumor and lymph nodes of vulvar cancer and characterized by an activation phenotype with stemness and good effector function in response to tumor or antibody stimulation. Therefore, as a promising new therapeutic strategy for vulvar tumor, the clinical application of TIL therapy should be further investigated for vulvar cancer patients.

\section{Acknowledgments}

This work was supported by the National Natural Science Foundation of China (81572575), Natural Science Foundation of Guangdong Province (2016A020215059), Natural Science Foundation of Guangdong Province (2021A1515010267), and Beijing Xisike Clinical Oncology Research Foundation (Y-2019 AZMS-0393). This institution has no role in the design of the study, collection, analysis, interpretation of data and writing of the manuscript.

\section{Author contributions}

T.Y., Z.S. and Y.L. conceived and designed the study, carried out experiments, and analyzed and interpreted data. Y.W. and H.Z. provided human samples and assisted with experiments. Z.L. and Y.L designed and supervised the study and data analysis. Z.S., D.Z., Y.C. and J.S. wrote the manuscript, with all authors 
contributing to writing and providing feedback.

\section{Disclosure statement}

Z.S., J.S., D.Z., and Y.L. were employed by the company Grit Biotechnology Co., Ltd. The remaining authors declare that the research was conducted in the absence of any commercial or financial relationships that could be construed as a potential conflict of interest.

\section{Availability of data and materials}

The datasets used and/or analyzed during the current study are available from the corresponding author on reasonable request.

Figures

A.
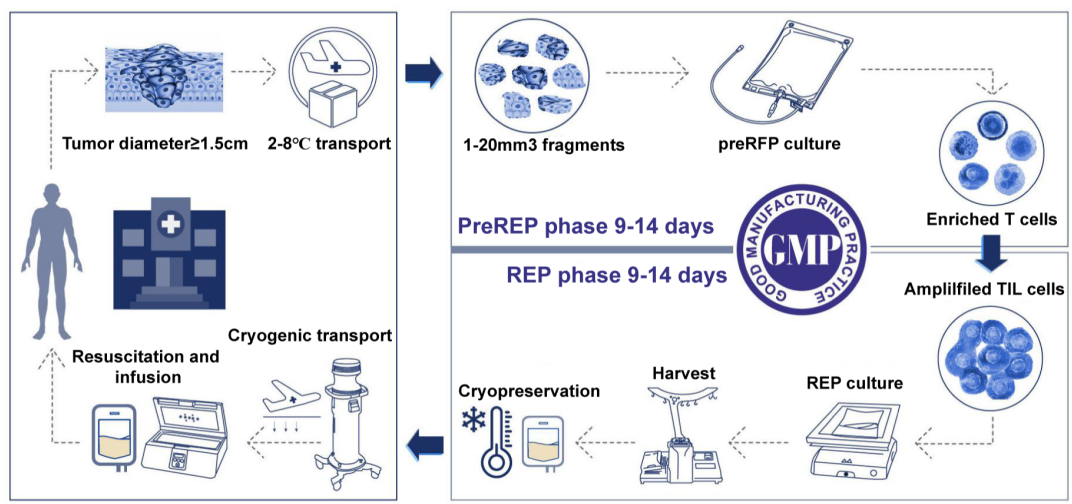

B.
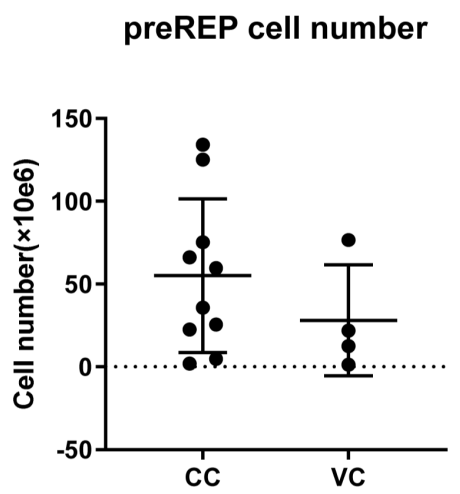

C.

REP expansion

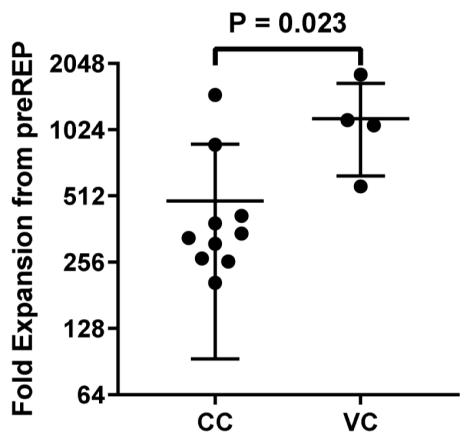

Figure 1.TIL can be successfully expanded from cervical and vulvar cancer. (A). A schematic representation of the TIL preparation process. (B). The total number of TILs expanded from cervical or vulvar tumor fragments was measured at the end of preREP culture. Each point represents the total TIL number generated from the tumor specimen of an individual patient. (C). The fold expansion of each TIL batch from preREP to the end of REP. 
A.

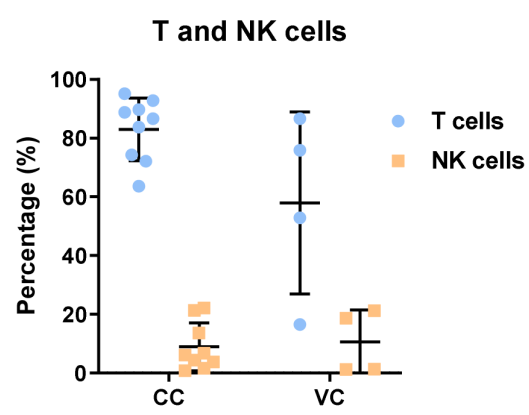

C.

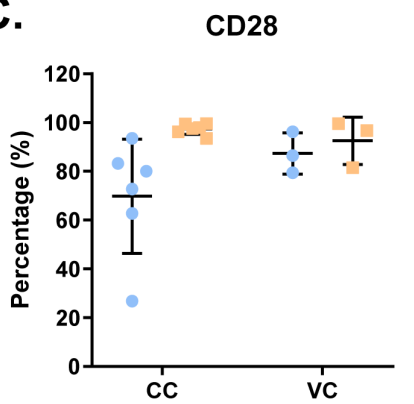

B.

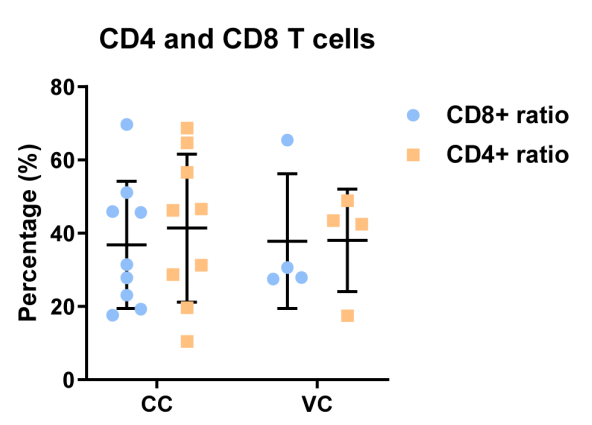

D.

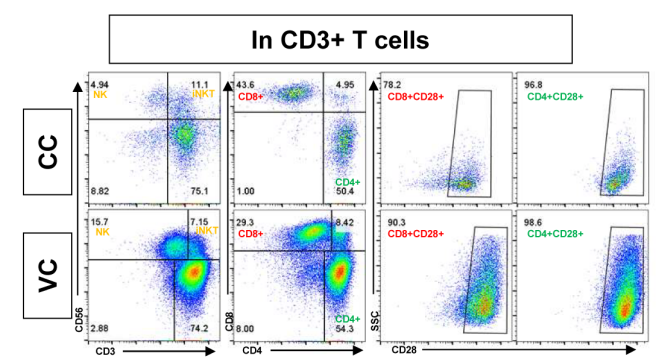

- CD8+ T cells $\quad$ CD4+ T cells

Figure 2. Profiling of CC and VC TILs after preREP. (A-B). At the end of preREP culture, TILs were collected from each batch and the percentage of $\mathrm{CD} 3^{+} \mathrm{T}$ cells, CD $4^{+} \mathrm{T}$ cells, $\mathrm{CD} 8^{+} \mathrm{T}$ cells, and CD $3^{-}$CD $56^{+}$NK cells was measured by flow cytometry. (C). CD28 expression on $\mathrm{CD}^{+}{ }^{+}$and $\mathrm{CD} 8^{+} \mathrm{T}$ cells was measured by flow cytometry.(D). Representative flow chart for each marker expressed. 

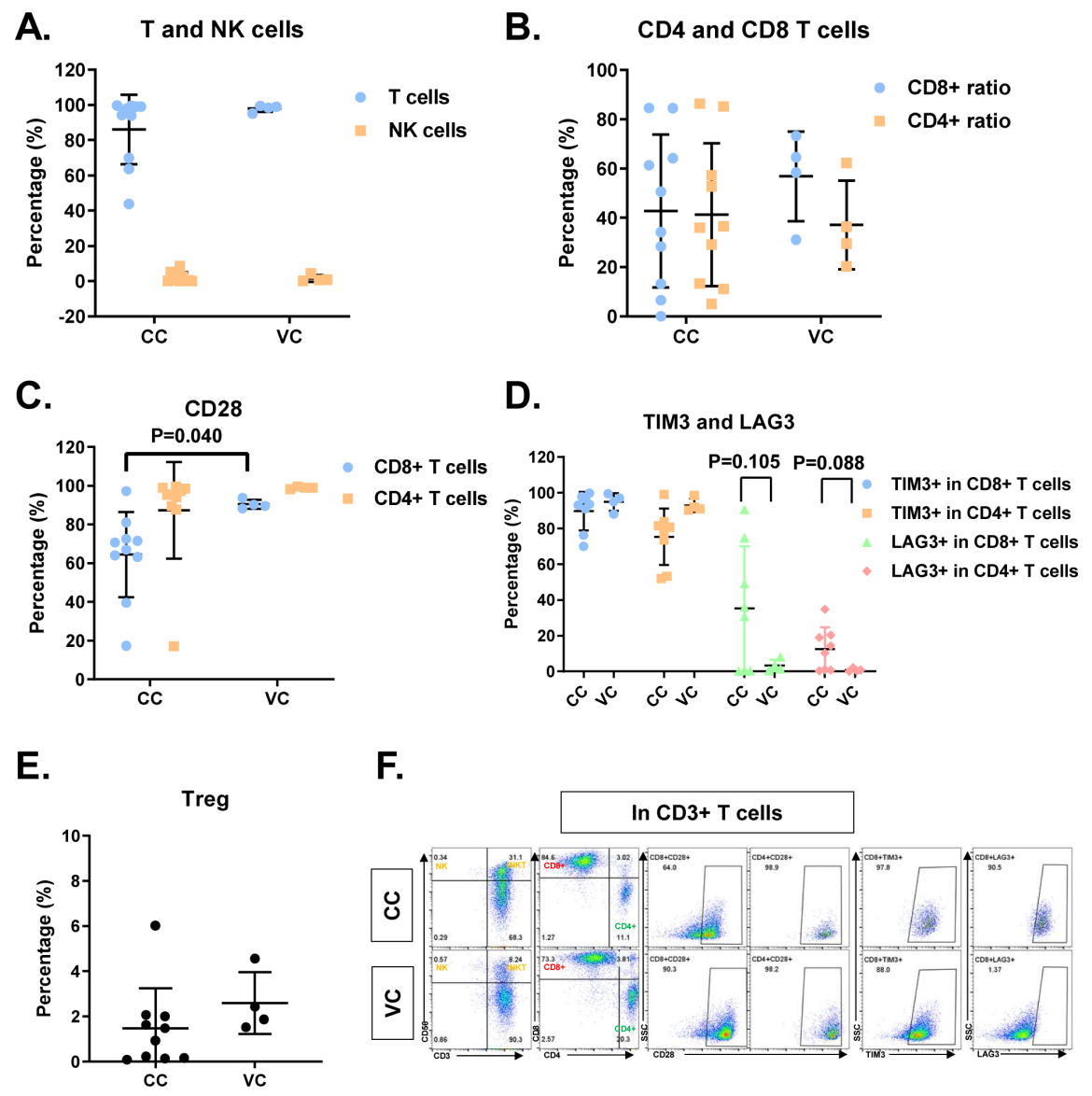

Figure 3.Profiling of CC and VC TILs at the end of REP. (A-B). At the end of REP culture, TILs were collected from each batch and the percentage of $\mathrm{CD}^{+} \mathrm{T}$ cells, $\mathrm{CD} 4^{+} \mathrm{T}$ cells, $\mathrm{CD} 8^{+} \mathrm{T}$ cells, and $\mathrm{CD}^{-}{ }^{-} \mathrm{CD} 56^{+} \mathrm{NK}$ cells was measured by flow cytometry. (C). CD28 expression on CD4 ${ }^{+}$and $\mathrm{CD} 8^{+} \mathrm{T}$ cells was measured by flow cytometry. (D). TIM3 and LAG3 expression on CD4 ${ }^{+}$and CD8 ${ }^{+}$T cells was measured by flow cytometry. (E). Treg ratio in TILs was analyzed with CD25 and Foxp3 staining by flow cytometry. (F). Representative flow chart for each marker expressed. 
A.

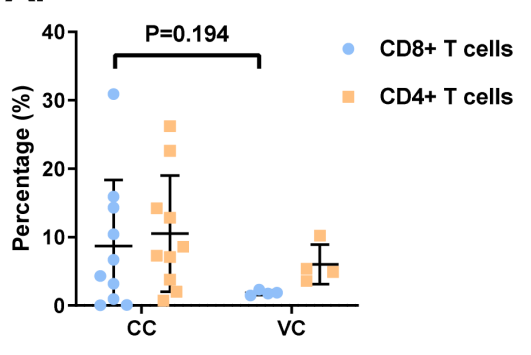

C.

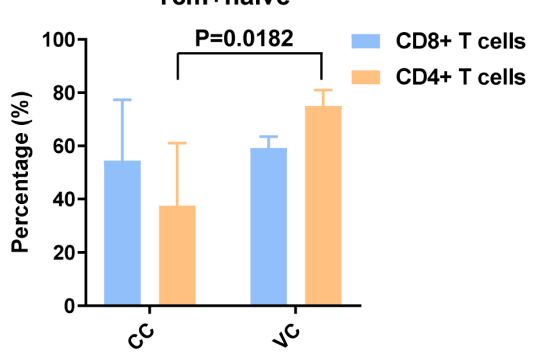

E.

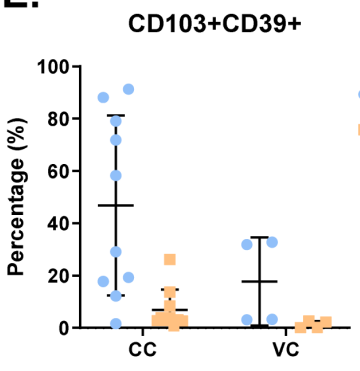

B.

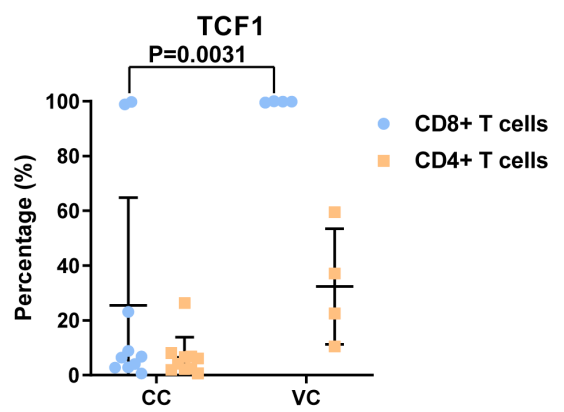

D.

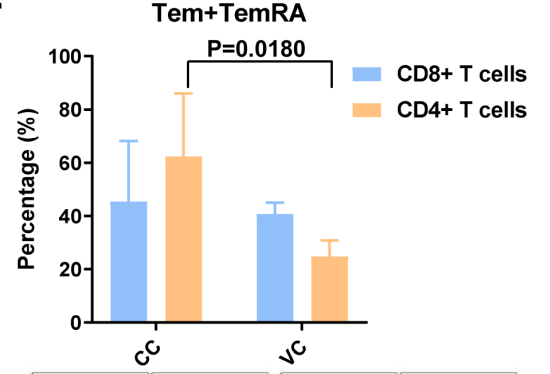

F.

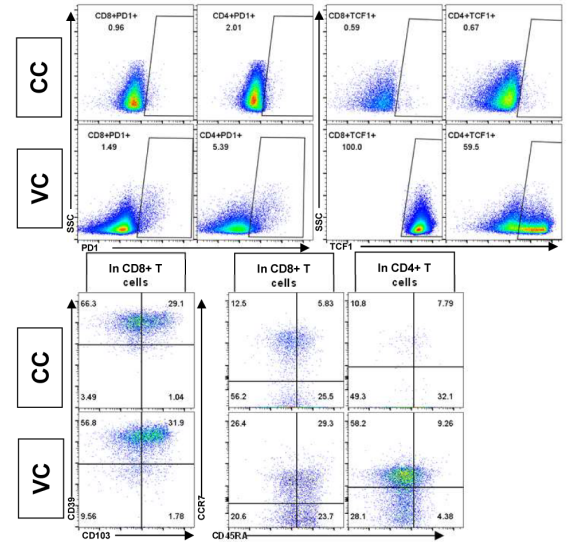

Figure 4. Stemness and memory phenotype analysis of CC and VC TILs. TILs were analyzed for the expression of indicated markers using flow cytometry after 9-14 days of rapid expansion. (A-B). The percentage of PD1 or TCF1 expression in TILs of each batch is shown. (C-D). The percentage of different $\mathrm{T}$ cell memory phenotypes is shown. Nä̈ve, central memory, effector memory and effector memory RA $\mathrm{T}$ cells are defined by $\mathrm{CD} 45 \mathrm{RA}^{+} \mathrm{CCR}^{+}, \mathrm{CD}^{+} 5 \mathrm{RA}^{-} \mathrm{CCR} 7^{+}, \mathrm{CD}^{-} 5 \mathrm{RA}^{-} \mathrm{CCR} 7^{-}$and $\mathrm{CD} 45 \mathrm{RA}{ }^{+} \mathrm{CCR} 7^{-}$, respectively.(E). The percentage of CD103 and CD39 expression in TILs of each batch is shown. (F). Representative flow chart for each marker expressed. 


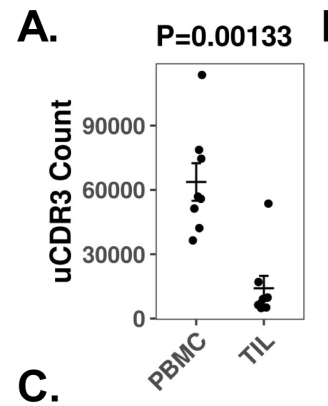

B.
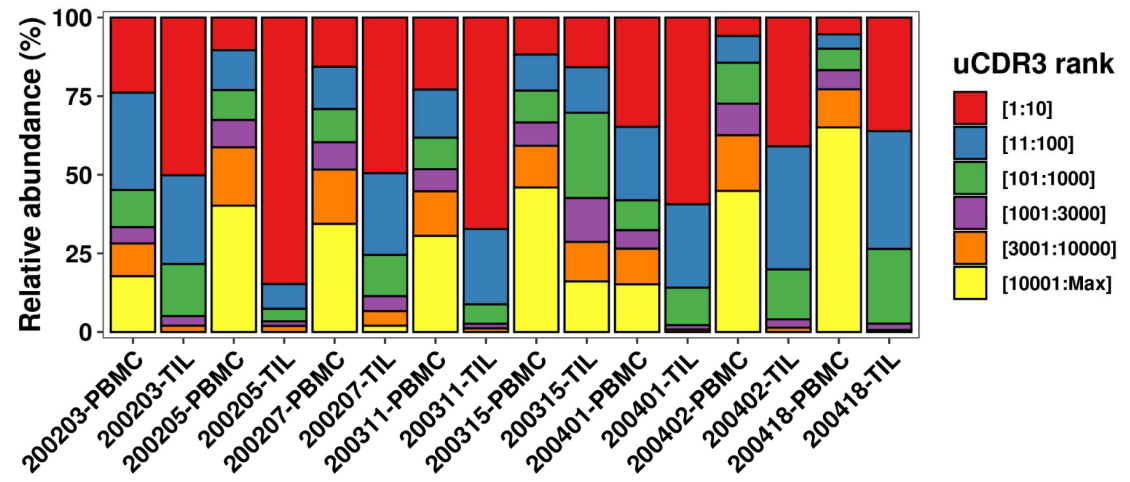

D.

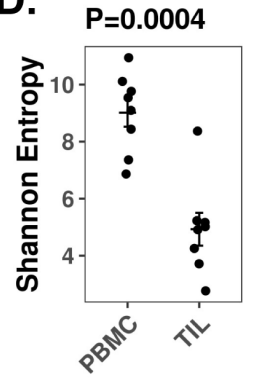

E.

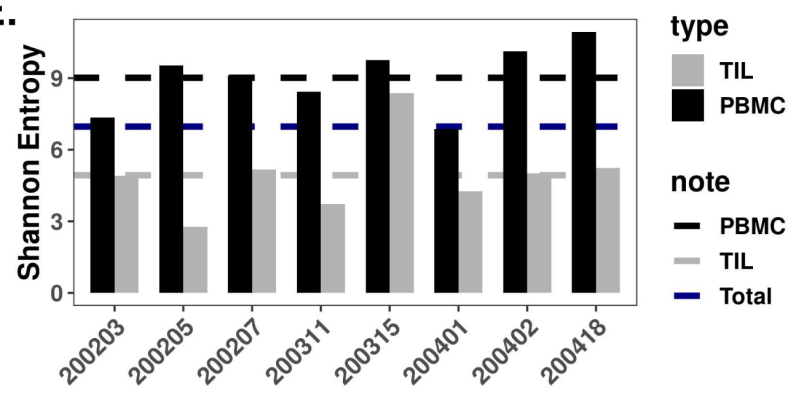

Figure 5. uCDR3 diversity characterization. (A). Unique CDR3 comparison between PBMC and TIL. Paired t test P-value was 0.00133.(B). Unique CDR3 counts in each sample. Mean uCDR3 counts of PBMC, TIL and total samples were shown in colored dash lines as indicated. (C). Percentage stacked barplot by sample. Colored bars indicate uCDR3 in different rank ordered by uCDR3 clone abundance. For example, red bar shows the total percentage of the Top10 ranked uCDR3 in each sample. (D). Shannon Entropy comparison between PBMC and TIL. Paired t test P-value was 0.0004. (E). Shannon Entropy in each sample. Mean Shannon Entropy of PBMC, TIL, and total samples were shown in colored dash lines as indicated. 


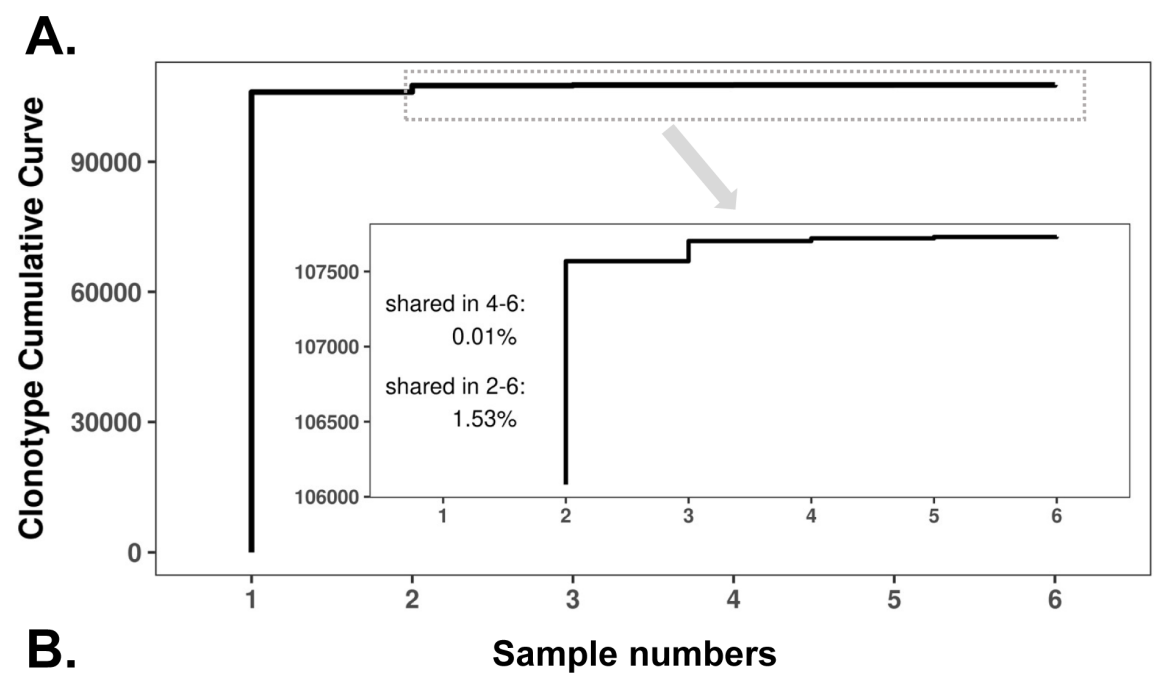

\begin{tabular}{|r|r|r|}
\hline $\begin{array}{r}\text { Clonotype Reads } \\
\text { Fraction }\end{array}$ & $\begin{array}{r}\text { Clonotype } \\
\text { Number }\end{array}$ & Percent (\%) \\
\hline $0 \%$ to $0.001 \%$ & 85251 & 38.896 \\
\hline $0.001 \%$ to $0.01 \%$ & 19986 & 9.119 \\
\hline $0.01 \%$ to $0.1 \%$ & 3644 & 1.663 \\
\hline $0.1 \%$ to $1 \%$ & 602 & 0.275 \\
\hline $1 \%$ to $10 \%$ & 95 & 0.043 \\
\hline $10 \%$ to $20 \%$ & 7 & 0.003 \\
\hline$>20 \%$ & 3 & 0.001 \\
\hline
\end{tabular}

Figure 6. Counts and frequency distribution of clonotypes. (A).Detection times of each clonotype in TIL samples. $\mathrm{X}$ axis indicates the number of samples having the same clonotype. $\mathrm{Y}$ axis indicates the cumulative number of clonotypes. Clonotypes were determined by the combination of CDR3 sequence, $\mathrm{V}$ gene and $\mathrm{J}$ gene. The cumulative number of shared clonotypes were highlighted in the zoomed subplot. Clonotypes detected in more than one TIL samples were considered as shared clonotypes. $1.53 \%$ clonotypes were detected in more than one TIL samples, and $0.01 \%$ clonotypes were detected in 4-6 TIL samples.(B). Reads fraction of shared clonotypes is shown as table. The reads fraction ranges at which these shared clonotypes were represented in their respective TIL samples were summarized in the left column. The clonotype number in each category and their corresponding percentage in total clonotype numbers were shown in the middle and right column, respectively. 
A.

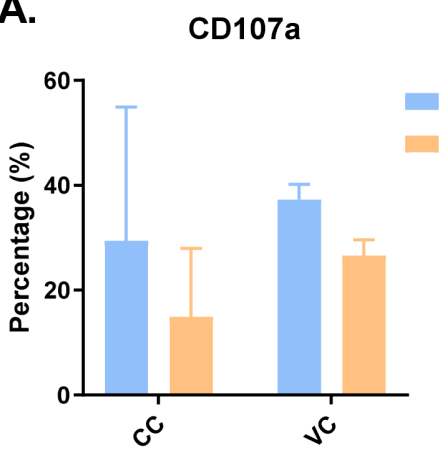

C.

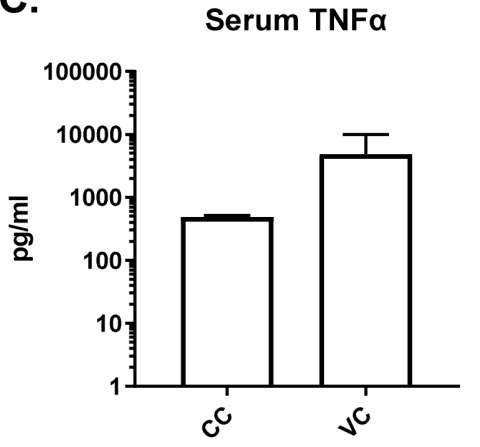

B.

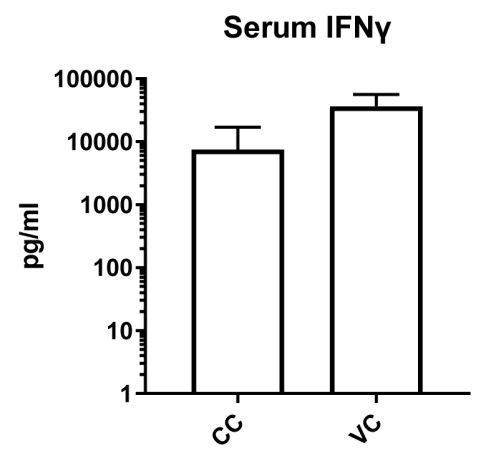

D.

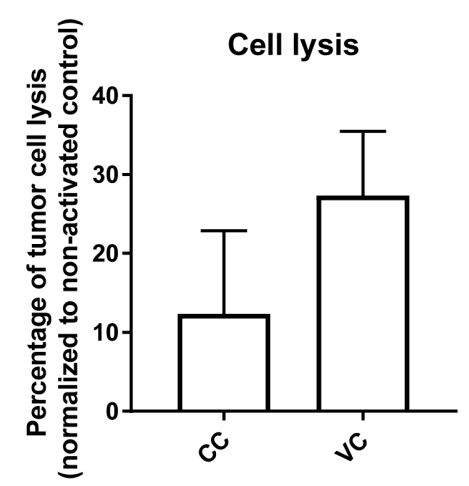

E.

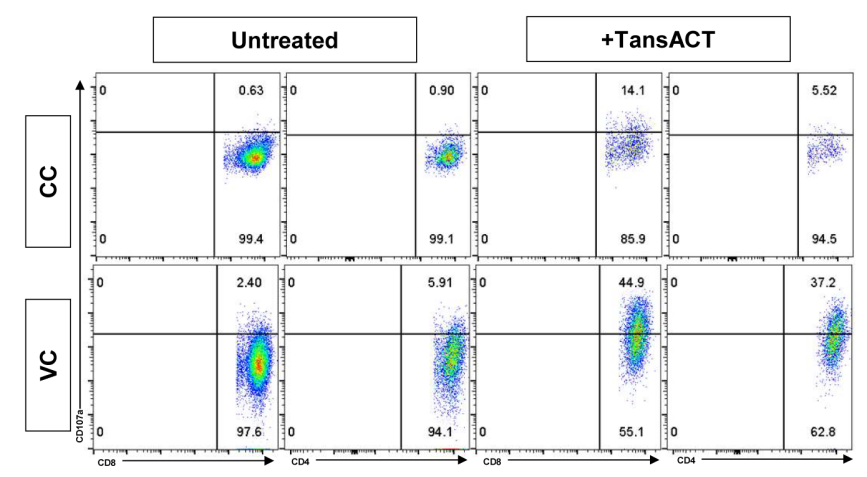

Figure 7.Functional characterization of CC and VC TILs. TILs were assessed for cytokine secretion and tumor killing capacity after rapid expansion. (A). TILs were re-stimulated by antibodies and CD107a expression was analyzed by flow cytometry. (B-C). IFN- $\gamma$ and TNF- $\alpha$ were detected by CBA in the supernatants of TIL after co-culture with HeLa cells line with an effector to target ratio of 3:1 overnight. Mean of IFN- $\gamma$ and TNF- $\alpha$ level $(\mathrm{pg} / \mathrm{ml})$ is shown above in each bar. (D). HeLa cells were labeled with CFSE before co-culturing with TILs. Cell lysis was monitored by flow cytometry after $18 \mathrm{hs}$. Tumor killing capacity is shown by the percentage of tumor cell lysis in the co-culture. (E). Representative flow chart for CD107a expressed.

Tables:

Table 1. Sample information.

\begin{tabular}{lllll}
\hline Sample\# & Tumor Types & Source of Tumor & Tumor Size & FIGO stage \\
\hline 1 & Cervical Caner & Primary & $0.5 \mathrm{~g}$ & IB1 \\
2 & Cervical Caner & Primary & $0.4 \mathrm{~g}$ & IIIC1
\end{tabular}




\begin{tabular}{lllll}
\hline Sample\# & Tumor Types & Source of Tumor & Tumor Size & FIGO stage \\
\hline 3 & Cervical Caner & Primary & $1.5 \mathrm{~g}$ & IB3 \\
4 & Cervical Caner & Primary & $1 \mathrm{~g}$ & IIB \\
5 & Cervical Caner & Primary & $1 \mathrm{~g}$ & IB2 \\
6 & Cervical Caner & Primary & $1 \mathrm{~g}$ & IB1 \\
7 & Cervical Caner & Primary and LN & $1.5 \mathrm{~g}$ & IIIC \\
8 & Cervical Caner & Primary & $0.9 \mathrm{~g}$ & IIIC1p \\
9 & Cervical Caner & Primary and LN & $2.9 \mathrm{~g}$ & IIIC2 \\
10 & Cervical Caner & Primary & $0.5 \mathrm{~g}$ & IB1 \\
11 & Vulvar Cancer & Primary & $0.7 \mathrm{~g}$ & IB \\
12 & Vulvar Cancer & LN & $0.5 \mathrm{~g}$ & IV \\
13 & Vulvar Cancer & Primary & $2.5 \mathrm{~g}$ & IIIA(i) \\
14 & Vulvar Cancer & Primary & $0.8 \mathrm{~g}$ & IB \\
\hline
\end{tabular}

\section{Reference}

1. Tan A, Bieber AK, Stein JA and Pomeranz MK: Diagnosis and management of vulvar cancer: A review. J Am Acad Dermatol 81: 1387-1396, 2019.

2. Survival rates for vulvar cancer. American cancer societyhttps://wwwcancerorg/cancer/vulvarcancer/detection-diagnosis-staging/survival-rateshtmlRevised on Feb 4th, 2019.

3. Couzin-Frankel J: Breakthrough of the year 2013. Cancer immunotherapy. Science 342: 1432-1433, 2013.

4. June CH, O'Connor RS, Kawalekar OU, Ghassemi S and Milone MC: CAR T cell immunotherapy for human cancer. Science 359: 1361-1365, 2018.

5. Nobbenhuis MA, Lalondrelle S, Larkin J and Banerjee S: Management of melanomas of the gynaecological tract. Curr Opin Oncol 26: 508-513, 2014.

6. Sznurkowski JJ, Zawrocki A, Emerich J and Biernat W: Prognostic significance of CD4+ and CD8+ T cell infiltration within cancer cell nests in vulvar squamous cell carcinoma. Int J Gynecol Cancer 21: 717-721, 2011.

7. Kortekaas KE, Santegoets SJ, Abdulrahman Z, et al : High numbers of activated helper T cells are associated with better clinical outcome in early stage vulvar cancer, irrespective of HPV or p53 status. J Immunother Cancer 7: 236, 2019.

8. Geukes Foppen MH, Donia M, Svane IM and Haanen JB: Tumor-infiltrating lymphocytes for the treatment of metastatic cancer. Mol Oncol 9: 1918-1935, 2015.

9. Bedognetti D, Spivey TL, Zhao Y, et al : CXCR3/CCR5 pathways in metastatic melanoma patients treated with adoptive therapy and interleukin-2. Br J Cancer 109: 2412-2423, 2013.

10. Mikucki ME, Fisher DT, Matsuzaki J, et al : Non-redundant requirement for CXCR3 signalling during tumoricidal T-cell trafficking across tumour vascular checkpoints. Nat Commun 6: 7458, 2015.

11. Rosenberg SA, Packard BS, Aebersold PM, et al : Use of tumor-infiltrating lymphocytes and interleukin2 in the immunotherapy of patients with metastatic melanoma. A preliminary report. N Engl J Med 319: $1676-1680,1988$.

12. Creelan B, Wang C, Teer J, et al : Abstract CT056: Durable complete responses to adoptive cell transfer using tumor infiltrating lymphocytes (TIL) in non-small cell lung cancer (NSCLC): A phase I trial. Cancer Research 80: CT056-CT056, 2020.

13. Tran E, Robbins PF, Lu YC, et al : T-Cell Transfer Therapy Targeting Mutant KRAS in Cancer. N Engl J Med 375: 2255-2262, 2016. 
14. Zacharakis N, Chinnasamy H, Black $\mathrm{M}$, et al : Immune recognition of somatic mutations leading to complete durable regression in metastatic breast cancer. Nat Med 24: 724-730, 2018.

15. Jazaeri AA, Zsiros E, Amaria RN, et al : Safety and efficacy of adoptive cell transfer using autologous tumor infiltrating lymphocytes (LN-145) for treatment of recurrent, metastatic, or persistent cervical carcinoma. Journal of Clinical Oncology 37: 2538-2538, 2019.

16. Bolotin DA, Poslavsky S, Mitrophanov I, et al : MiXCR: software for comprehensive adaptive immunity profiling. Nature Methods 12: 380-381, 2015.

17. Powell DJ, Jr., Dudley ME, Robbins PF and Rosenberg SA: Transition of late-stage effector T cells to CD27+ CD28+ tumor-reactive effector memory T cells in humans after adoptive cell transfer therapy. Blood 105: 241-250, 2005.

18. Datar I, Sanmamed MF, Wang J, et al : Expression Analysis and Significance of PD-1, LAG-3, and TIM-3 in Human Non-Small Cell Lung Cancer Using Spatially Resolved and Multiparametric Single-Cell Analysis. Clin Cancer Res 25: 4663-4673, 2019.

19. Jansen CS, Prokhnevska N, Master VA, et al : An intra-tumoral niche maintains and differentiates stem-like CD8 T cells. Nature 576: 465-470, 2019.

20. Chen L and Han X: Anti-PD-1/PD-L1 therapy of human cancer: past, present, and future. J Clin Invest 125: 3384-3391, 2015.

21. Siddiqui I, Schaeuble K, Chennupati V, et al : Intratumoral Tcf1(+)PD-1(+)CD8(+) T Cells with Stem-like Properties Promote Tumor Control in Response to Vaccination and Checkpoint Blockade Immunotherapy. Immunity 50: 195-211 e110, 2019.

22. Utzschneider DT, Charmoy M, Chennupati V, et al : T Cell Factor 1-Expressing Memory-like CD8(+) T Cells Sustain the Immune Response to Chronic Viral Infections. Immunity 45: 415-427, 2016.

23. Duhen T, Duhen R, Montler R, et al : Co-expression of CD39 and CD103 identifies tumor-reactive CD8 T cells in human solid tumors. Nat Commun 9: 2724, 2018.

24. Ribas A and Wolchok JD: Cancer immunotherapy using checkpoint blockade. Science 359: 1350-1355, 2018.

25. Gros A, Parkhurst MR, Tran E, et al : Prospective identification of neoantigen-specific lymphocytes in the peripheral blood of melanoma patients. Nature medicine 22: 433-438, 2016.

26. Crane CA, Han SJ, Ahn B, et al : Individual patient-specific immunity against high-grade glioma after vaccination with autologous tumor derived peptides bound to the $96 \mathrm{KD}$ chaperone protein. 19: 205-214, 2013.

27. Turcotte S, Gros A, Hogan K, et al : Phenotype and function of T cells infiltrating visceral metastases from gastrointestinal cancers and melanoma: implications for adoptive cell transfer therapy. J Immunol 191: 2217-2225, 2013. 